\title{
Unveiling the Multi-wavelength Phenomenology of Anomalous X-ray Pulsars
}

\author{
GianLuca Israel ${ }^{1}$, Luigi Stella ${ }^{1}$, Stefano Covino $^{2}$, Sergio Campana ${ }^{2}$, \\ Lorella Angelini ${ }^{3}$, Roberto Mignani ${ }^{4}$, Sandro Mereghetti ${ }^{5}$, Gianni \\ Marconi $^{6}$, Rosalba Perna ${ }^{7}$ \\ ${ }^{1}$ INAF - Osservatorio Astronomico di Roma, Italy \\ ${ }^{2}$ INAF - Osservatorio Astronomico di Brera, Italy \\ ${ }^{3}$ NASA/Goddard, Space Flight Center, USA \\ ${ }^{4}$ European Southern Observatory, Garching, Germany \\ ${ }^{5}$ CNR, Istituto di Astrofisica Spaziale e Fisica Cosmica, Milano, Italy \\ ${ }^{6}$ European Southern Observatory, Paranal, Chile \\ ${ }^{7}$ Princeton University, Princeton, USA
}

\begin{abstract}
During 2002-2003 the number of IR-identified counterparts to the Anomalous X-ray Pulsars (AXPs) has grown to four $(4 \mathrm{U} 0142+61$, 1E 2259+586, 1E 1048.1-5937 and RXS J170849-400910) out of the six objects (plus two candidates) known in this class. More importantly, some new common characteristics have been identified, such as IR variability, IR flattening in the broad-band energy spectrum, X-ray spectral variability as a function of pulse phase (which are not predicted by the magnetar model), and X-ray bursts (which cannot be explained in terms of standard accretion models). We present the results obtained from an extensive multi-wavelength observational campaign carried out with the NTT and CFHT for the optical/IR bands, and XMM and Chandra (plus BeppoSAX archival data) in X-rays. Based on these results and those reported in the literature, the IR-to-X-ray emission of AXPs is compared.
\end{abstract}

\section{Introduction}

It is now commonly believed that Soft Gamma-ray Repeaters (SGRs) are magnetars - neutron stars powered by decay of their strong magnetic fields $(B>$ $10^{14}$ gauss). AXPs have been linked to SGRs because of similarly large periods $(5<P<12 \mathrm{~s})$ and period derivatives (Thompson \& Duncan 1993, 1996). What differentiates these two seemingly dissimilar sets of objects is, at present, unclear. Nonetheless, there is a growing group of radio pulsars (Camilo et al. 2000) with similarly long periods and with inferred magnetic field strengths approaching $10^{14} \mathrm{G}$. These pulsars possess no special attributes linking them to either the AXPs (Pivovaroff, Kaspi \& Camilo 2000) or to the SGRs. Thus periodicity alone does not appear to be a sufficient attribute for classification. Conversely, a very high magnetic field strength cannot be the sole factor governing whether or not a neutron star is a magnetar, a radio pulsar or in a binary system. One possibility is that AXPs and SGRs are linked temporally. Specifically, three out 
of the six AXPs are associated with supernova remnants (SNRs) whereas only SGR 0526-66 has a plausible SNR association. Taken at face value, these data suggest that AXPs evolve into SGRs, but this hypothesis has severe problems, in that as a class $P_{\mathrm{SGR}} \sim P_{\mathrm{AXP}}$ and $B_{\mathrm{SGR}} z B_{\mathrm{AXP}}$ (Kulkarni et al. 2003).

The recent detection of X-ray bursts from 1E 2259+586 and 1E 1048.1-5937 has strengthened the possible connection of AXPs with SGRs (Kaspi \& Gavriil 2002; Gavriil, Kaspi \& Woods 2002; Kaspi, these proceedings). In the case of $1 \mathrm{E} 2259+586$, IR variability of the likely counterpart was detected a few days after strong X-ray bursting activity. Also, the variability of the IR counterpart to $1 \mathrm{E} 1048.1-5937$ is thought to be related to X-ray variability (Israel et al. 2002). Although these properties open a new horizon in the field, we do not yet understand what specific physical parameter(s) differentiate AXPs from SGRs.

Evidence for flattening (or excess) of the IR flux, compared to a simple blackbody component extrapolated from the X-ray data, has been reported in four AXPs: 1E 2259+586, 1E 1048.1-5937, 4U 0142+61 and RXS J170849400910 (Hulleman et al. 2001; Wang \& Chakrabarty 2002; Israel et al. 2003). The magnetar scenario does not account for the observed IR emission or variability in AXPs and no predictions can therefore be verified. On the other hand, the accretion models fail to account for the bursts. Therefore, none of the proposed theoretical models (at least in their present form) seem to be able to account simultaneously for the IR, optical and X-ray emission of AXPs. In this respect the IR emission from AXPs/SGRs may play a key role in their understanding.

\section{Chandra HRC-I and IR Adaptive Optics Observations}

The IR-to-X-ray data we relied upon have been obtained as part of a joint ESO/Chandra HRC-I project. About $10 \mathrm{ks}$ of Chandra observations have been obtained for RXS J170849-400910 and 1E 1048.1-5937, while 20 ks have been devoted to AX J1845-0258. Optical/IR data have been obtained for all known AXPs with the 3.5-m New Technology Telescope (NTT, La Silla, Chile; SOFI and SUSI2 instruments), the 4-m Canada France Hawaii Telescope (CFHT with OAB), and the Very Large Telescope (VLT with FORS and ISAAC).

Israel et al. $(2002,2003)$ have already reported the identification of the likely IR counterpart of RXS J170849-400910, and the detection of IR variability from the counterpart of $1 \mathrm{E} 1048.1-5937$.

$4 \mathrm{U} 0142+61$ was observed at CFHT in the $J, H$ and $K^{\prime}$ bands with a source PSF of $\sim 0^{\prime} ! 16$, from which we obtained $J=22.3 \pm 0.1, H=21.1 \pm 0.1$ and $K^{\prime}=20.0 \pm 0.1$ (Israel et al., in preparation). Figure 1 (left) summarizes the photometric results: the IR counterpart stands out in the color-color diagram $\left(J-K^{\prime}=2.3\right)$ with respect to the other objects in the field.

$K^{\prime}$-band observations were also carried out for $1 \mathrm{E} 2259+586$ at the CFHT, two months after the detection of SGR-like burst activity from the pulsar (Kaspi $\&$ Gavriil 2002). Also in this case, thanks to the use of adaptive optics and PSF $\sim 0^{\prime}$. 19, we detected the IR counterpart at $K^{\prime}=21.7 \pm 0.2$ (about 0.4 magnitudes brighter than reported by Hulleman et al. 2001; see also Fig. 1, center). This implies that after two months, the IR "activity" of $1 \mathrm{E} 2259+586$ is still present.

Based on the above results and those in the literature, we present the broadband energy spectra of the four AXPs with known IR/optical counterparts in 

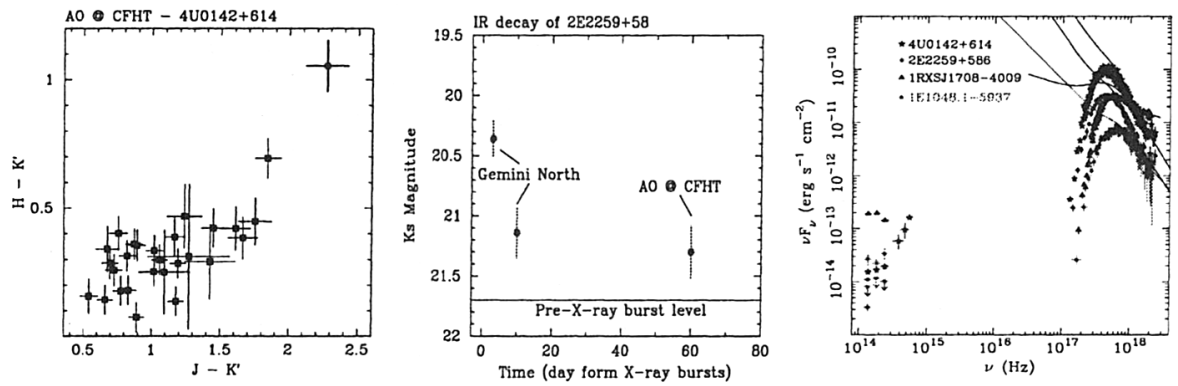

Figure 1. Left panel: IR color-color diagram obtained for the region (radius of $30^{\prime \prime}$ ) around the position of $4 \mathrm{U} 0142+61$, based on observations carried out on 2002 Aug from the CFHT. The counterpart (in the upper right corner) clearly stands out with respect to the other objects in the field. Center panel: IR decay "lightcurve" of 1E $2259+586$ inferred by using the CFHT data presented here and those in the literature (Kaspi et al. 2003). Right panel: Broad-band energy spectrum of AXPs. X-ray raw data are taken from the medium and low energy instruments on board BeppoSAX while the solid upper curves are the unabsorbed fluxes for the blackbody plus power law models. On the lower left corner of the plot are the optical/IR unabsorbed fluxes.

Figure 1, (right). The scatter in both unabsorbed X-ray and IR fluxes is large. We also note that RXS J170849-400910 is the AXP which most deviates from the other sources in both bands. Finally, the IR fluxes of $4 \mathrm{U} 0142+61$ presented here show a deviation from the blackbody component originally used to fit the optical fluxes (Hulleman, van Kerkwijk \& Kulkarni 2000). Given the extremely high values of extinction in the direction of the AXPs, the flux ratio $F_{X} / F_{I R}$ might be quite different depending on the assumed X-ray energy range.

\section{The Debated Case of AX J1845-0258}

AX J1845-0258 has been a candidate AXP since the discovery of $7 \mathrm{~s}$ X-ray pulsations with $A S C A$ (Torii et al. 1998; Gotthelf \& Vasisht 1998). One year later the source was about 10 times fainter, and no periodicity was detected (Vasisht et al. 2000). Therefore, its identification as a possible AXP was based merely on the period and spectral parameters of the source in the "high" level.

In order to clarify the nature of AX J1845-0258 we obtained BeppoSAX, Chandra and $X M M$ observations, and also optical/IR images from the NTT. In all X-ray images we detected only a faint object, the position and count rates of which were consistent with those of the second $A S C A$ observation. Figure 2 (left) shows the BeppoSAX and $X M M$ spectra of AX J1845-0258 which are well fitted by a highly absorbed $\left(N_{H} \sim 5 \times 10^{22} \mathrm{~cm}^{-2}\right)$ power law $(\Gamma=1.2 \pm 0.8)$ or alternatively by a $1.0 \pm 0.5 \mathrm{keV}$ blackbody. In both cases the unabsorbed luminosity is in the $10^{33}-10^{34} \mathrm{ergs} \mathrm{s}^{-1}$ range (assuming distances of 7 and $15 \mathrm{kpc}$ ). The optical/IR observations revealed no peculiar objects within the Chandra $1^{\prime \prime}$ uncertainty region down to $H \approx 21$. 

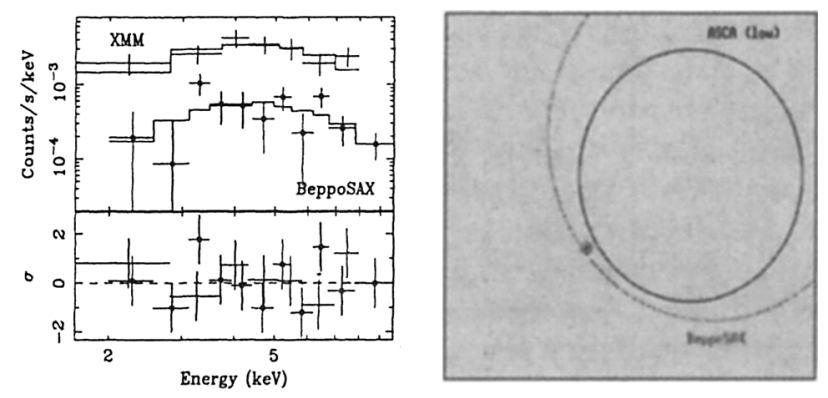

Figure 2. Left panel: XMM EPIC and BeppoSAX MECS spectra of AX J1845-0258 fitted with an absorbed power law model. Right panel: The Chandra HRC-I image is shown superimposed with the $A S C A$ and $B e p p o S A X$ positional uncertainty regions at the $90 \%$ confidence level.

Acknowledgments. This work is supported by CNAA, ASI, CNR and Ministero dell'Università e Ricerca Scientifica e Tecnologica (MURST) grants. The authors thank Olivier Hainaut, Leonardo Vanzi and the NTT team for their kind help during ESO observations. We also thank Olivier Lai and the CFHT team for their support, and Hank Donnelly of the Chandra team.

\section{References}

Camilo, F., Kaspi, V. M., Lyne, A. G., Manchester, R. N., Bell, J. F., D'Amico, N., McKay, N. P. F., \& Crawford, F. 2000, ApJ, 541, 367

Gavriil, F. P., Kaspi, V. M., \& Woods, P. M. 2002, Nature, 419, 142

Gotthelf, E. V., \& Vasisht, G., 1998, New Astronomy, 3, 293

Hulleman, F., van Kerkwijk, M. H., \& Kulkarni, S. R. 2000, Nature, 408, 689

Hulleman, F., Tennant, A. F., van Kerkwijk, M. H., Kulkarni, S. R., Kouveliotou, C., \& Patel, S. K. 2001, ApJ, 563, L49

Israel, G. L., et al. 2002, ApJ, 580, L143

- 2003, ApJ, 589, L93

Kaspi, V. M., \& Gavriil, F. P. 2002, IAU Circ. 7924

Kaspi, V. M., Gavriil, F. P., Woods, P. M., Jensen, J. B., Roberts, M. S. E., \& Chakrabarty, D. 2003, ApJ, 588, L93

Kulkarni, S. R., Kaplan, D. L., Marshall, H. L., Frail, D. A., Murakami, T., \& Yonetoku, D. 2003, ApJ, 585, 948

Pivovaroff, M. J., Kaspi, V. M., \& Camilo, F. 2000, ApJ, 535, 379

Thompson, C., \& Duncan, R. C. 1993, ApJ, 408, 194

- 1996, ApJ, 473, 322

Torii, K., Kinugasa, K., Katayama, K., Tsunemi, H., \& Yamauchi, S. 1998, ApJ, 503,843

Vasisht, G., Gotthelf, E. V., Torii, K., \& Gaensler, B. M. 2000, ApJ, 542, L49

Wang, Z., \& Chakrabarty, D. 2002, ApJ, 579, L33 